Meta

Journal des tradlucteurs

Translators' Journal

\title{
Les dictionnaires de terminologie linguistique : bibliographie systématique
}

\section{P. Swiggers et M. Janse}

Volume 36, numéro 4, décembre 1991

URI : https://id.erudit.org/iderudit/004312ar

DOI : https://doi.org/10.7202/004312ar

Aller au sommaire du numéro

Éditeur(s)

Les Presses de l'Université de Montréal

ISSN

0026-0452 (imprimé)

1492-1421 (numérique)

Découvrir la revue

Citer cet article

Swiggers, P. \& Janse, M. (1991). Les dictionnaires de terminologie linguistique :

bibliographie systématique. Meta, 36(4), 647-653.

https://doi.org/10.7202/004312ar d'utilisation que vous pouvez consulter en ligne. 


\section{Les dictionnaires de terminologie linguistique: bibliographie systématique}

Devant la multiplicité des théories et des modèles linguistiques, le débutant - mais en fait aussi la plupart des linguistes professionnels - reste un peu perplexe, étant incapable de maîtriser les différentes approches. Ce qui accroît la difficulté, c'est l'emploi de terminologies très diverses, reflétant d'une part les approches théoriques différentes, mais cachant d'autre part certaines convergences (dans le rapport entre théories et faits). Il n'existe pas - ni sous forme imprimée ni sous forme automatisée un «dictionnaire comparatif» de la terminologie linguistique, et il faut en premier lieu 
recourir aux quelques dictionnaires de terminologie linguistique qui ajoutent aux termes relevés et commentés des correspondants dans d'autres théories linguistiques, voire dans $\mathrm{d}^{\text {'autres traditions linguistiques }}{ }^{1}$. Si une uniformisation de la terminologie linguistique ${ }^{2}$ est aujourd'hui une vue de l'esprit, on peut toutefois souhaiter que s'installe une «métalexicographie» systématisante, prenant comme objet les unités théoriques du discours linguistique et visant à mettre en place les principes de base pour la traduction de ces termes techniques ${ }^{3}$.

Signalons d'emblée que la bibliographie qui suit ${ }^{4}$ ne prétend pas être exhaustive: nous avons inclus les ouvrages dont nous avons pris connaissance directe ou dont nous avons retrouvé le signalement bibliographique. La bibliographie, qui n'inclut pas les dictionnaires de langues - dans lesquels on trouvera parfois des indications précieuses sur l'histoire des termes grammaticaux ${ }^{5}-$ a été constitué en fonction d'une progression logique dans la démarche de consultation. Nous avons d'abord donné la bibliographie sur les dictionnaires de terminologie linguistique et ensuite les travaux qu'on consultera en corrélation directe avec la demande d'informations plus précises et complètes. L'utilisateur de dictionnaires de termes linguistiques peut consulter un dictionnaire de linguistique pour s'informer sur le sens d'un terme (et sur son équivalent dans la langue-source ou la langue-cible pertinente pour l'utilisateur), ou il peut recourir au dictionnaire pour situer certains termes, rencontrés lors de lectures, dans le cadre théorique où ils s'insèrent. Enfin, la consultation du dictionnaire de termes linguistiques peut servir à des recherches sur l'origine et l'évolution de certaines notions linguistiques, ou sur l'articulation conceptuelle d'une théorie. Ces buts sont en général servis par des ouvrages terminologiques de nature très différente: nous pensons, dans l'ordre respectif, aux «glossaires» (contrastifs) de termes - signalant un terme linguistique et son équivalent dans une autre langue (celle du public visé) - , aux lexiques généraux ${ }^{6}$, présentant des définitions concises de termes linguistiques, aux lexiques spécialisés (terminologie d'un auteur ou d'une école, avec renvois bibliographiques) et aux dictionnaires encyclopédiques de linguistique ${ }^{7}$, qui présentent des descriptions plus étendues de termes, avec intégration de la bibliographie pertinente ${ }^{8}$. Pour les sections 2-5, nous avons adopté un classement alphabétique.

\section{BIBLIOGRAPHIE GÉNÉRALE SUR LES DICTIONNAIRES DE TERMINOLOGIE LINGUISTIQUE}

Pour une bibliographie commentée mais assez sommaire de huit dictionnaires de terminologie linguistique, tous en allemand ${ }^{9}$, on se reportera au fascicule suivant:

BECKERS, Hartmut und Peter SCHMITTER (dir.) (1978): Kommentierte Übersicht über sprachwissenschaftliche Wörterbücher und allgemeine Einführungen (Studium Sprachwissenschaft, Heft 1), Münster, Institut für Allgemeine Sprachwissenschaft der Westfälischen Wilhelms-Universität.

Les articles suivants fournissent un état de la question (non exhaustif) de la lexicologie métalinguistique:

ACHMANOVA, O.S., A.I. POLTORACKUJ (1962): "Slovari lingvističeskoj terminologii", Leksikografičeskij Sbornik, 5, pp. 188-191.

KOERNER, E.F.K. (1972): "Glossaries of Linguistic Terminology, 1951-1971: An Overview”, Linguistische Berichte, 18, pp. 30-38.

HARTMANN, R.R.K. (1972): "More on Glossaries of Linguistic Terminology", Linguistische Berichte, 21, pp. 77-79. 
CONTE, Maria-Elisabeth (1973): "Dizionari e glossari di terminologia linguistica: 1950-1973", Studi di Grammatica Italiana, 3, pp. 273-287.

KREUDER, Hans-Dieter (1973): "Lexika der modernen linguistischen Terminologie", Zeitschrift für Dialektologie und Linguistik, 40, pp. 175-184.

BUSSMANN, Hadumod (1974): "Lexika der sprachwissenschaftlichen Terminologie. Bericht über eine vernachlässigte Gattung (I)", Deutsche Sprache 1974, pp. 43-66.

BUSSMANN, Hadumod, ALTMANN, Hans und Hartmut LAUFFER (1975): "Lexika der sprachwissenschaftlichen Terminologie. Bericht über eine vernachlässigte Gattung (II)", Deutsche Sprache 1975, pp. 147-172; 255-269.

SIMECEK, Václav (1975): "Slovníky lingvistické terminologie" [Dictionnaires de terminologie linguistique], Cizi Jazyky ve Skole (Prague), 18, pp. 399-409; 451-456.

HARTMANN, Dietrich (1976): "Neuere linguistische Wörterbücher", Studium Linguistik 2, pp. 54-65.

FETZER, Wilfried (1981): "Bibliographie sprachwissenschaftlicher Wörterbücher: eine Ergänzung zu Koerner und Hartmann" (Ling. Berichte, Heft 18 und 21, 1972), Wiesbaden, Vieweg.

On trouvera un utile inventaire de termes linguistiques dans le catalogue suivant:

DUTZ, Klaus D., KACZMAREK, Ludger und Hans J. WULFF (1983): Systematischer Katalog der Allgemeinen Sprachwissenschaft. Überarbeitete und ergänzte Fassung. Mit Registern, Münster, Institut für Allgemeine Sprachwissenschaft \& MAKS Publikationen [c.r. de P. Swiggers dans Leuvense Bijdragen, 74, 1985, pp. 539-541.]

Pour les problèmes de terminologie linguistique, on pourra consulter :

JACOBSSON, G. (dir.) (1980): Aktual'nye problemy po lingvističeskoj terminologii [Problèmes actuels de la terminologie linguistique], Göteborg.

VASCENCU, V. (1975): Probleme de terminologie lingvistică [Problèmes de terminologie linguistique], București.

Pour des considérations théoriques sur l'emploi de la terminologie grammaticale ou

linguistique en didactique des langues, on pourra se reporter aux deux recueils suivants :

LEROT, Jacques et Jean KLEIN (1984): Terminologie grammaticale. Essai de clarification et d' harmonisation, Bruxelles, De Boeck.

BAUSCH, Karl-Heinz und Siegfried GROSSE (1987): Grammatische Terminologie in Sprachbuch und Unterricht, Düsseldorf, Schwann, (Sprache der Gegenwart, 69).

\section{GLOSSAIRES (CONTRASTIFS) DE TERMINOLOGIE LINGUISTIQUE}

ALEXANDRU, T. si R. CHIVESCU et al. (1978): Dicţionar poliglot de termeni lingvistici [Dictionnaire polyglotte de termes linguistiques], București, Tipografia Univ. din Bucureşti.

ALI AL KHULI, M. (1982): A Dictionary of Theoretical Linguistics. English-Arabic with an Arabic-English Glossary, Beirut, Librairie du Liban.

ANGHELESCU, Nadia (1988): Mic dicționar român-arab de termeni lingvistici, București, Tipografia Univ. din București.

ARUNABHARATHI, N. (1976): Glossary of Linguistics English-Tamil, Madras, Tamil Nuulagam.

ARUTJUNJANC [HAROWT JOWNJANC], Michail A. (1963): Armjano-russkij lingvističeskij slovar', Erevan.

BAKALLA, M.H., AL-KASIMI, Ali M., AL-RAYYIH, M.K., SAAD, G.N. and M.E. SIENY (1983): A Dictionary of Modern Linguistic Terms. English-Arabic \& Arabic-English, Beirut, Librairie du Liban.

BALOUN, Jaroslav (1982): Némecko-český slovník lingvistické terminologie. / Deutschtschechisches Wörterbuch der linguistischen Terminologie, Prague, Státní pedag. naklad, [2 volumes parus: A-Bedeutungen].

BARAKÉ, B. (1985): Dictionnaire de linguistique. Français-arabe, avec un index alphabétique des termes arabes, Tripoli, Jarrouss Press.

Bhäshätața paribhäshä-kosha [Terminologie de la linguistique] (1970): Dacca, Central Board for Development of Bengali.

CACHIA, Pierre (1973): The Monitor. A dictionary of Arabic grammatical terms. Arabic-English./EnglishArabic, Beirut, Librairie du Liban, London, Longman.

GOWDA, Kempe K. (Éd.) (1976): Bhashavijnana kosha [Glossaire de termes linguistiques], Mysore, Institute of Kannada Studies. 
GRÖSCHEL, Bernhard und Elena PARWANOWA (1985): Russisch-Deutsches Wörterbuch der linguistischen Terminologie, Münster, Institut für Sprachwissenschaft der Westfälischen Wilhelms-Univ., 2 volumes (Studium Sprachwissenschaft, Beiheft 3, 1-2).

HATIBOĞLU, V. (1972): Dilbilgisi terimleri sözlüğ̈̈ [Glossaire de termes linguistiques], s.1.

KENESBAEV, S. i T. ŽANUZAKOV (1966²): Russko-kazachskij slovar' lingvističeskich terminov [Dictionnaire russe-kazakh de termes linguistiques], Alma-Ata, Nauka.

KIELSKI, Bolesław (1959): Stownik terminologii gramatycznej francuskiej i polskiej [Dictionnaire de termilologie grammaticale française et polonaise], Zódź, Zakład im. Ossolińskich [Z/odzkie Towartzystwo Naukowe, Wydzial I, 35].

MIJINGUINI, Abdou (1983): Vocabulaire technique des sciences du langage (français-hausa), Niamey, Organisation de l'Unité africaine/Centre d'études linguistiques et historiques par tradition orale.

MAN, O., L. KOVAL et al. (1960): Rusko-český slovník lingvistické terminologie [Dictionnaire russe-tchèque de terminologie linguistique], Prague, Státní pedag. naklad.

NASH, R. (Éd.) (1968): Multilingual Lexicon of Linguistics and Philology. English, Russian, German, French, Coral Gables (FL), University of Miami Press.

PATERNOST, J. (1965): Russian-English Glossary of Linguistic Terms, University Park, Pennsylvania State University.

PATERNOST, J. (1966): Slovenian-English Glossary of Linguistic Terms, University Park, Pennsylvania State University.

Sāhitya ane b̆hāshāvijñānani paribhashā [Terminologie de littérature et de linguistique], s.d. Amadāvāda, Gujarāta Univarsit̀̀.

SAKTHIVEL, S. (1980): Moliyiyal kalaiccollakaräti cucaktivēl [Glossaire des termes techniques de linguistique], Annamalainagar, Meena Pathippakam.

SCHMALSTIEG, W.R. and A. KLIMAS (1971): Lithuanian-English Glossary of Linguistic Terminology, University Park, Pennsylvania State University.

VARDAR, B., GÜZ, N., GÜZELŞEN, M.R., ÖZTOKAT, E., O. SENEMOĞLU, (1978): Bașlica dilbilim terimleri [Glossaire de termes de linguistique], Istanbul, Ist. Üniversitesi Yayinlari.

VASCENCU, Victor (dir.) (1981): Dicționar român-rus de termeni lingvistici și filologici [Dictionnaire roumain-russe de termes linguistiques et philologiques], București, Tipografia Univ. din București.

\section{LEXIQUES GÉNÉRAUX ${ }^{10}$ DE LA LINGUISTIQUE}

ACHMANOVA, O.S. (dir) (1966) : Slovar' lingvističeskich terminov [Dictionnaire de termes linguistiques], Moscou, Izd. Sovetskaja Ënciklopedija, $\left[196^{2}\right.$; trad. anglaise: 1977 , Linguistic Terminology, Moscou, Moscow University Press].

BOOIJ, G.E., KERSTENS, J.G., H.J. VERKUYL (1975): Lexicon van de taalwetenschap [Dictionnaire de la linguistique], Utrecht-Antwerpen, Spectrum, [19802].

BORBA, Francisco da Silva (19762): Pequeno vocabulário de linguistica moderna, São Paulo, Companhia Editora Nacional.

CÂMARA, Joaquim Mattoso, Jr. (1956) : Dicionário de fatos gramaticais, Rio de Janeiro, Casa de Rui Barbosa (Coleção de Estudios Filológicos, 1); 19642, Dicionário de filologia e gramática, referente à lingua portuguesa $; 1968^{3} ; 1977^{8}$ : Dicionário de linguistica e gramatica, Petropolis, Vozes; $1981^{9} \mathrm{]}$.

CARDONA, Giorgio Raimondo (1988): Dizionario di linguistica, Roma, Armando Editore.

CARRETER, Fernando Lázaro (1953): Diccionario de términos filológicos, Madrid, Gredos, [19622; $1968^{3}$ avec réimpressions en 1971 et 1974].

CONRAD, R. (1975): Kleines Wörterbuch sprachwissenschaftlicher Termini, Leipzig, VEB Bibliographishes Institut, $\left[1985^{2}\right.$, Lexikon sprachwissenschaftlicher Termini $]$.

CONSTANTINESCU-DOBRIDOR, Gheorghe (1980): Mic dicționar de terminologie lingvistică [Petit dictionnaire de terminologie linguistique], București, Albatros.

Dicionário gramatical (1953): Rio de Janeiro, Editôra Globo.

DUBOIS, Jean, GIACOMO, Mathée, GUESPIN, Louis, MARCELLESI, Christiane, MARCELLESI, JeanBaptiste et Jean-Pierre MÉVEL (1973): Dictionnaire de linguistique, Paris, Larousse [19742; trad. italienne, 1979, Bologna, Zanichelli; trad. esp. 1979, Madrid, Alianza Editorial].

GENTILE, Aniello (1963): Lessico di terminologia linguistica, Napoli, Liguori.

GERSON, Stanley (1969): A Glossary of Grammatical Terms. An aid to the student of languages, St. Lucia, University of Queensland Press.

GOKAB, Zbigniew, HEINZ, Adam, Kazimierz POLAŃSKI (1968): Stownik terminologii językoznawczej [Dictionnaire de la terminologie linguistique], Warszawa, Prástwowe wydawnictwo naukowe, [19702].

HANIC , D.I., I.S. OLINYYK (1985): Slovnyk linhvistychich terminiv [Dictionnaire de termes linguistiques], Kiev, Vyšc̆a s̆kola. 
HAKULINEN, Auli, Jussi OJANEN (1970): Kielitieteen ja fonetiikan termistöä [Terminologie de linguistique et de phonétique], Forssa (Suomalaisen Kirjallisuuden Seuran Toimituksia, 324) [1976²].

HARTMANN, R.R.K. and F.C. STORK (1972): Dictionary of Language and Linguistics, London, Applied Science Publishers Ltd.

HELBIG, G. (1969): Kleines Wörterbuch linguistischer Termini, Leipzig, Herder-Institut [suppl. 2 à Deutsch als Fremdsprache 6/1969].

HEUPEL, Carl (1973): Taschenwörterbuch der Linguistik, München, List Verlag, [1978³, Linguistisches Wörterbuch, München, Deutscher Taschenbuch-Verlag/List].

HOFFMANN, J.B. und H. RUBENBAUER (1950): Wörterbuch der grammatischen und metrischen Terminologie, Heidelberg, C. Winter, [19632].

HORECKÝ, Ján, Anna RÁCOVÁ (1979): Slovník jazykovedných termínov Dictionnaire de termes linguistiques], Bratislava, Slov. pedag. naklad.

JOTA, Zélio dos Santos (1976): Dicionário da lingüística, Rio de Janeiro, Presença [19812].

JUREVIC, A.L. (1962): Slovar' lingvističeskich terminov [Dictionnaire de termes linguistiques], Minsk, Izd. Min-va vyšs

KROTEVIC̆, Je.V., N.S. RODZEVIČ (1957): Slovnyk lingvistychych terminov, [Dictionnaire de termes linguistiques], Kiev, Akad. Nauk URSR.

MAROUZEAU, J. (1933): Lexique de la terminologie linguistique; français, allemand, anglais, italien, Paris, P. Geuthner, [19432; 19513, avec des réimpressions en 1961 et 1969; trad. russe: 1960, Slovar' lingvističeskich terminov].

MEETHAM, A.R. and R.A. HUDSON (Eds.) (1969): Encyclopaedia of Linguistics, Information, and Control, Oxford-London, Pergamon Press.

MOUNIN, Georges (dir.) (1974): Dictionnaire de la linguistique, Paris, PUF.

NILSSON, Stig (1977): Språkliga termer [Termes linguistiques], Stockholm, Natur och kultur.

PECIAR, Štefan et al. (1952): Zakládná jazykovedná terminologia [Terminologie linguistique fondamentale], Bratislava, Slov. Akad. Vied a Umení.

PEI, Mario (1966): Glossary of Linguistic Terminology, New York - London, Columbia University Press [éd. de poche: Garden City, Doubleday, 1966] [19692].

PEI, Mario A. and Frank GAYNOR (1954): A Dictionary of Linguistics, New York, Philosophical Library [19672, Totowa, Littlefield, Adams \& Co]

PETROSYAN, H.Z., GALSTYAN, S.A., Tc.A. LARAGYOWLYAN (1975): Lezvabanakan báaran [Dictionnaire linguistique], Erevan, Haykakan SSH Gitowtcyownneri Akad. hratarakčcowtcyown [titre parallèle en russe: PETROSJAN, G.Z., GALSTJAN, S.A. et T.A. KARAGULJAN: Lingvističeskij slovar'].

PHÉLIZON, J.-F. (1976): Vocabulaire de la linguistique, Paris, Roudil.

ROZENTAL, D.É., M.A. TELENKOVA (1972): Spravočnik lingvističeskich terminov [Répertoire de termes linguistiques], Moscou, Prosveščenie, [1976²; 1985³].

SEVERINO, A. (1937): Manuale di nomenclatura linguistica, Milano, Ed. "Le lingue estere".

SIMEON, R. (1969): Enciklopedijski rječnik lingvističnih naziva na 8 jezika (hrvatsko-srpski, latinski, ruski, njemacki, engleski, francuski, talijanski, španjolski) [Dictionnaire encyclopédique de termes linguistiques en 8 langues: serbo-croate, latin, russe, allemand, anglais, français, italien, espagnol], Zagreb, Matica Hrvatska, 2 volumes.

SIMONE, Raffaele (1969): Piccolo dizionario della linguistica moderna, Torino, Lascher.

SJÖGREN, Peter A. (1979): Termer $i$ allmän språkvetenskap. Ett systematiskt lexicon [Termes de linguistique générale. Lexique systématique], Stockholm, Akademilitteratur.

SPRINGHETTI, Aemilius (1962): Lexicon linguisticae et philologiae, Roma, Apud Pontificiam Universitatem Gregorianam.

STEIBLE, Daniel J. (1967): Concise Handbook of Linguistics: A Glossary of Terms, New York, Philosophical Library, London, P. Owen.

ULRICH, Winfried (1972): Wörterbuch: linguistische Grundbegriffe, Kiel, Ferdinand Hirt.

VARDAR, B., GÜZ, N., ÖZTOKAT, E., RIFAT, M., SENEMOĞLU, O., E.SÖZER (1980): Dilbilim ve dilbilgisi terimleri sözlüğ̈̈, Ankara, Sevinç Basimevi.

VERMEER, Hans J. (1971): Einführung in die linguistische Terminologie, München, Nymphenburger Verlagshandlung; Darmstadt, Wissenschaftliche Buchgesellschaft.

WALSH, Donald D. (1963): What's What: A List of Useful Terms for the Teacher of Modern Languages, New York, Modern Language Association.

\section{LEXIQUES SPÉCIALISÉS : THÉORIES ET DOMAINES DE RECHERCHE}

ABAD, Francisco (1986): Diccionario de lingüística de la escuela española, Prólogo de R. Lapesa, Madrid, Gredos. 
AMBROSE-GRILLET, Jeanne (1978): Glossary of Transformational Grammar, Rowley (Mass.), Newbury House Publ.

BÉCARES-BOTAS, Vicente (1985): Diccionario de terminología gramatical griega, Salamanca, Universidad de Salamanca.

ENGLER, Rudolf (1968): Lexique de la terminologie saussurienne, Utrecht-Anvers, Spectrum ${ }^{11}$.

DE FELICE, Emidio (1954): La terminologia linguistica di G.I. Ascoli e della sua scuola, Utrecht-Anvers, Spectrum.

HAMP, Eric P. (1957): A Glossary of American Technical Linguistic Usage 1925-1950, Utrecht-Antwerp, Spectrum, $\left[1963^{2} ; 1966^{3} ; 1968^{4}\right.$; trad. russe : 1964, Slovar' amerikanskoj lingvističeskoj terminologii].

JEDLICKKA, Alois (dir.) (1977-1979): Slovník slovanské lingvistické terminologie. / Slovar' slavjanskoj lingvističeskoj terminologii./Dictionary of Slavonic linguistic terminology, Prague, Academia/Hamburg, Buske, 2 volumes.

LANG, Ewald (1967): Terminologie der generativen Grammatik/Terminology of Generative Grammar/Terminologija porozdajušcej grammatiki, Berlin, Deutsche Akademie der Wissenschaften (DDR), [Ronéot].

NARVESON, Bert H. (1952): Functional Grammar Terms for Language Students, Northfield (Minnesota), St. Olaf College Press.

PALMATIER, Robert A. (1972): A Glossary for English Transformational Grammar, New York, Meredith Corporation.

SÁNCHEZ DÍAZ, Aníbal y Ernesto ZIERER (1971): Glosario explicativo inglés-castellano de términos de gramática generativa transformacional, Trujillo, Universidad nacional.

Slavjanska lingvistična terminologija [Terminologie linguistique slave], Sofija, Izd. ba BAN.

VACHEK, Josef et Josef DUBSKY (1960): Dictionnaire de linguistique de l'École de Prague, Utrecht-Anvers, Spectrum, $\left[1966^{2} ; 1970^{3} ;\right.$ trad. russe: 1964$]$.

\section{DICTIONNAIRES ENCYCLOPÉDIQUES DE LA LINGUISTIQUE}

ABRAHAM, Werner (1974): Terminologie zur neueren Linguistik, Zusammengestellt von W. Abraham unter Mitwirkung von R. Elema, R. Griesen, A.P. ten Cate und J. Kok, Tübingen, M. Niemeyer, [trad. espagnole : 1981, Madrid, Gredos; $1988^{2}$ édition révisée et augmentée, en 2 volumes].

BOHUSCH, Otmar (1972): Lexikon der grammatischen Terminologie. Zusammenstellung und Erklärung des deutschen, englischen, französischen und lateinischen Vokabulars zur Sprachlehre und Sprachkunde, Donauwörth, L. Auer.

BUSSMANN, Hadumod (1983): Lexikon der Sprachwissenschaft, Stuttgart, A. Kröner.

CRYSTAL, David (1980): A Dictionary of Linguistics and Phonetics, Oxford, B. Blackwell, London, A. Deutsch, [19852].

DUCROT, Oswald et Tzvetan TODOROV (1972): Dictionnaire encyclopédique des sciences du langage, Paris, Seuil, [traduction allemande: 1975, Enzyklopädisches Wörterbuch der Sprachwissenschaften, Frankfurt am Main, Athenaion, trad. anglaise: 1981, Encyclopaedic Dictionary of the Sciences of Language, Oxford, B. Blackwell].

KNOBLOCH, Johann (Éd.) (1961): Sprachwissenschaftiches Wörterbuch, Heidelberg, C. Winter (2 volumes parus: A-F).

LEWANDOWSKI, Theodor (1973): Linguistisches Wörterbuch, Heidelberg, Quelle \& Meyer, 3 tomes, [19752 ; $\left.1980^{3} ; 1984-1985^{4}\right]$.

MARTINET, André (dir.) (1969): La linguistique: Guide alphabétique, avec la collaboration de Jeanne Martinet et d'Henriette Walter, Paris, Denoël.

POTTIER, Bernard (dir.) (1973): Le langage, Paris, Centre d'étude et de promotion de la lecture.

SCHMITT, Alfred (1933): Probe eines Wörterbuchs der sprachwissenschaftlichen Terminologie, den Sprachwissenschaftlern zur Begutachtung unterbreitet, Vorwort von Leo Weisgerber, Berlin-Leipzig, de Gruyter, [donne le texte d'un article-spécimen: "Akzent"].

STAMMERJOHANN, Harro (unter Mitarbeit von Hildegard JANSSEN) (1975): Handbuch der Linguistik. Allgemeine und angewandte Sprachwissenschaft, München, Nymphenburger Verlagshandlung.

WELTE, Werner (1974): Moderne Linguistik. Terminologie/Bibliographie. Ein Handbuch und Nachschlagewerk auf der Basis der generativ-transformationellen Sprachtheorie, München, M. Hueber, 2 tomes.

P. SWIGGERS ET M. JANSE Fonds national belge de la recherche scientifique (N.F.W.O.)! Bibliographie linguistique (La Haye) 


\section{NOTES}

1. Voir surtout les dictionnaires de Knobloch (1961-) et d'Abraham (1974,19882), cf. section 5.

2. Rappelons qu'un tel projet - conçu dans une visée d'une linguistique ne décrivant que les faits observables - sous-tendait la contribution de Leonard Bloomfield à l'International Encyclopedia of Unified Science, cf. L. Bloomfield, Linguistic Aspects of Science, Chicago, 1939 et voir H. Hiz - P. Swiggers, "Bloomfield, the Logical Positivist", Semiotica, $\mathrm{n}^{\circ} 79,1990$, pp. 257-270.

3. Signalons aussi la latitude et l'incertitude qui règnent dans la traduction des termes désignant les différentes langues du monde.

4. La présente bibliographie est un remaniement (avec ajout de plus de 30 titres) de celle publiée par $P$. Swiggers dans L'Information grammaticale 40, 1989, pp. 12-15.

5. Comme un modèle dans son genre on peut mentionner l'ensemble des articles grammaticaux rédigés par Henri Bonnard dans le Grand Larousse de la langue française, Paris, 1971-1978 (7 volumes).

6. Les appellations sont arbitraires en ce sens qu'elles ne correspondent pas (toujours) aux titres des ouvrages. Les distinctions faites sont basées sur notre jugement personnel. La bibliographie inclut des ouvrages de terminologie grammaticale et linguistique au sens général. Nous avons exclu les dictionnaires qui concernent de façon exclusive une branche particulière (par ex. la phonétique, l'onomastique, etc.) et les ouvrages qui tout en étant consacrés aux problèmes de terminologie linguistique ne se présentent pas sous forme de glossaires ou de dictionnaires (encyclopédiques).

7. Nous y avons ajouté l'ouvrage de Ducrot et Todorov, dont le statut oscille entre celui d'un dictionnaire de linguistique et celui d'une encyclopédie de la linguistique, et les ouvrages collectifs dirigés par Martinet et Pottier.

8. Ce dernier critère nous semble fondamental pour décider du caractère «encyclopédique» du dictionnaire: dans la section 5 nous avons placé les dictionnaires qui intègrent la bibliographie citée (à la fin de chaque entrée ou dans une section bibliographique) dans la définition et la description des termes.

9. Il s'agit des dictionnaires suivants: Abraham (1974), Bohusch (1972), Conrad (1975), Heupel (1973), Knobloch (1961-), Lewandowski (1973), Ulrich (1972), et Welte (1974). Ces dictionnaires sont analysés aux pages $7-20$ du fascicule cité.

10. Pour une première orientation on consultera les ouvrages d'Achmanova (le meilleur travail de ce genre: très complet et bien informé), de Dubois, de Goląb, de Mounin ou de Simeon; pour la philologie au sens classique, on utilisera le dictionnaire de Springhetti.

11. Voir aussi: R. Godel, Les sources manuscrites du Cours de linguistique générale de $F$. de Saussure, Genève, Droz, 1969 (19571), p. 252-281: «Lexique de la terminologie», Rudolf Engler (communication personnelle) prépare un index de son édition critique (Wiesbaden, Harrassowitz, 1967-) du Cours de linguistique générale de F. de Saussure; cf. les spécimens présentés dans R. Engler, «Diachronie: l'apport de Genève» et «'Attività', 'atto', 'azione': considerazioni su una voce "marginale" dell'indice saussuriano», Cahiers Ferdinand de Saussure, ${ }^{\circ}$ 42, 1988, pp. 127-166 et 167-174. 